\title{
Background Updating with the Use of Intrinsic Curves ${ }^{\star}$
}

\author{
Joaquín Salas $^{1,2}$, Pedro Martínez ${ }^{1}$, and Jordi González ${ }^{3}$ \\ ${ }^{1}$ CICATA Querétaro-IPN \\ ${ }^{2} \mathrm{CVC}$ \\ ${ }^{3}$ UPC-CSIC
}

\begin{abstract}
A primary tool to extract information about moving objects is background subtraction. In this technique, the difference between a model of what is static, or background, and the current image of the scene gives information about what is in the prime plane or foreground. This study focus on the pixelwise updating mechanism of the background model throughout the analysis of the images provided by a fixed camera. The concept of intrinsic curves, early introduced in the field of stereovision, is extrapolated to the problem of detecting the moving boundaries. We use a mixture of Gaussians to register information about the recent history of the pixel dynamics. Our method improves this model in two ways. Firstly, it reduces the chances of feeding the mixture of Gaussians with foreground pixels. Secondly, it takes into account not just the scalar pixel value but a richer description of the pixel's dynamics that carries information about the interpixel variation. Ample experimental results in a wide range of environments, including indoors, outdoors, for a different set of illumination conditions both natural and artificial are shown.
\end{abstract}

\section{Introduction}

Nowadays, there is an increasing demand for automatic monitoring systems based on image analysis fueled by factors such as the increasing existence of imaging devices, our own limitations to watch every available video source, an the development of important multimedia applications [18]. Motion is a basic capability of visual perception systems. Many important higher perceptual tasks can be built on top of it, including tracking, and recognition. A primary tool to extract information about moving objects is background subtraction. In this technique, the difference between a background model and the current image of the scene gives information about what is in the prime plane or foreground. Depending on the definition of background, its general solution will involve the distinction of foreground objects even when the background objects are moving[17], and indeed, even when the camera itself is moving[1]. However, there is a fairly large number of scenarios where the constraint of a static background imaged with static cameras is rather useful and interesting applications can be developed.

Piccardi [16] proposes a classification of background construction methods based on speed, memory requirements and accuracy. Functionally, the problem can be divided

\footnotetext{
* This work has been partially supported by the SIP-IPN grant 20061072, and EC grant IST027110 for the HERMES project and by the Spanish MEC under projects TIC2003-08865 and DPI-2004-5414. J. González also acknowledges the support of a Juan de la Cierva Postdoctoral fellowship from the Spanish MEC.
} 


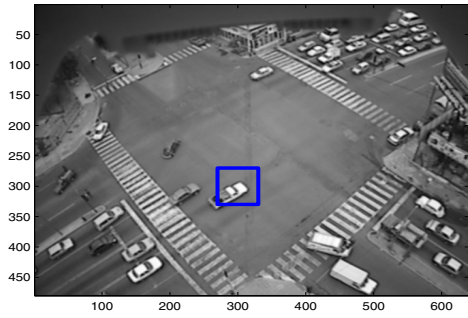

(a) A sample frame out of a test sequence. The blue square is detailed in (b).

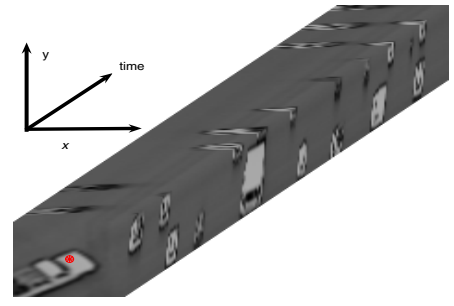

(b) Image patch intensity variations throughout time.

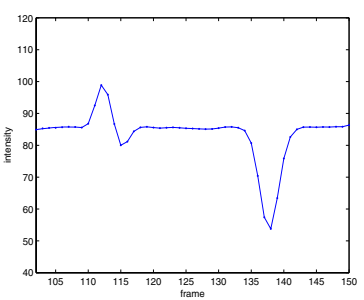

(c) Intensity.

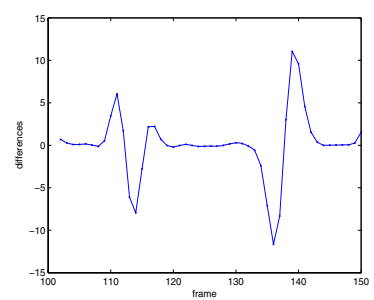

(d) Differences.

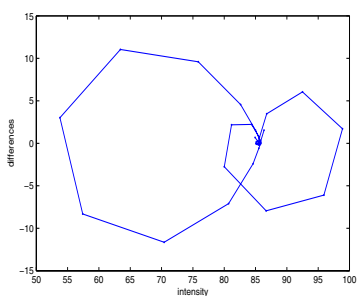

(e) Intensity versus Differences.

Fig. 1. A pixel is observed during 40 frames. The space-time representation of the image patch in (a) is shown in (b). The patch central pixel intensity variations(c) and differences(d), generate the intrinsic curve in (e).

into several interesting ones. Consider for instance, the initialization, or how to arrive to the initial background model. Since it may be seen as a classification problem some researchers have used optical flow to either formulate hypotesis[5], train Neural Networks[4], or use Support-Vector Machines [12]. Another problem is to reduce the time to figure out the background revealed by a moving foreground object. Kaup and Aach[10] exploit spatiotemporal correlation, and motion information. The surrounding known background area is analyzed and spatially extrapolated using an spectral domain extrapolation algorithm. These techniques are rather important for applications such as videoconferencing [13] where background coding for efficient compression and transmission is needed. The problem of updating the background model has many interesting facets. Since deciding a pixel classification based solely on the base of a single threshold may be too limiting, Kumar et al.[11] suggest the use of a hysteresis threshold technique. After a decision has been taken about what is background and what is foreground, there is still space for improving the results. Filling holes, dilating borders are among the usual strategies used. He et al.[7] present a background updating algorithm which combines the variation of a neighboring area with the difference between the current and previous values in order to predict the new values of the pixels on the background. Nowadays, it is widely accepted that color can be used to identify the shadows casted by objects[2]. Horprasert[8] designs a color model that separates the brightness from the chromaticity component. Their conclusion is similar to Han et al. [6] whom propose an algorithm to deal with gradual illumination changes. To cope with 


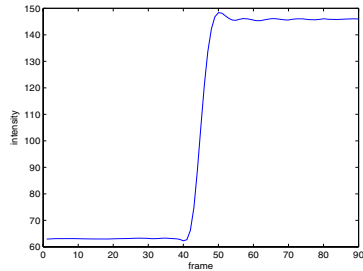

(a) Intensity.

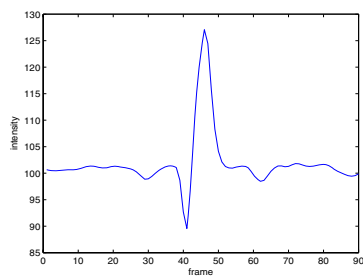

(d) Intensity.

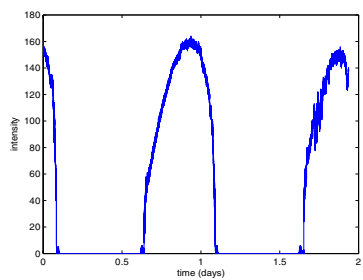

(g) Intensity.

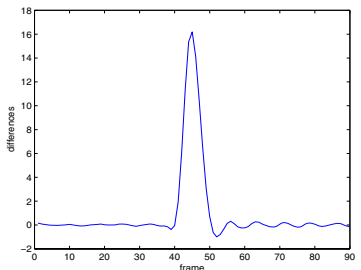

(b) Differences.

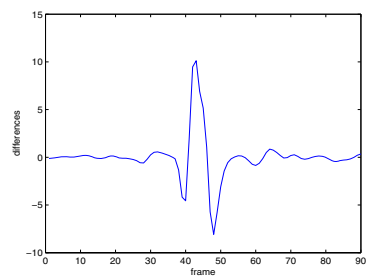

(e) Differences.

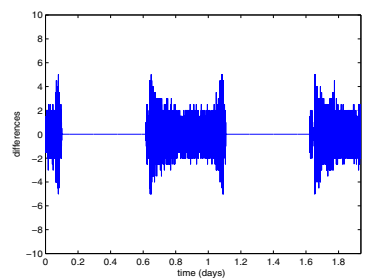

(h) Differences.

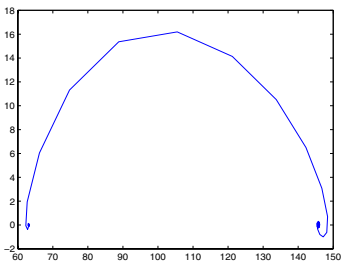

(c) Intensity versus Differences.

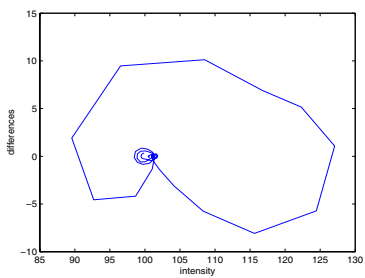

(f) Intensity versus Differences.

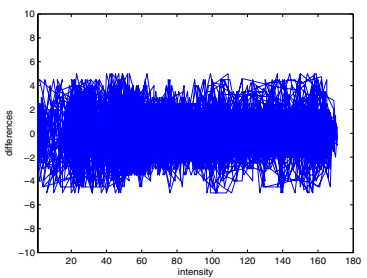

(i) Intrinsic curve.

Fig. 2. The different classes of intrinsic curves that can be observed. In (a)-(c) the stability zone moves from one place to another. In (d)-(f) the curve comes back to the original stability zone after the occluding object pass by. In (g)-(i) the stability zone extends smoothly on the intensity axis. The first two sets of figures correspond to a few dozens of frames. The last set corresponds to two days of observations of the same pixel.

the problem of shadows, they propose a color model where chromaticity distortion is measured.

In this paper, we introduce a technique for background updating that uses intrinsic curves[21]. Intrinsic curves are $N$-values functions whose components are obtained from applying operators $P_{n}$ to the intensity variation over time. This technique works at pixel level. It can be complemented with the knowledge generated at region level[25] or scene level[22]. The advantage of treating each pixel independently is that this gives a lot of flexibility at the expense of greater variability due to noise. For cases where the illumination conditions are controlled and there is the opportunity for observing the scene without foreground objects a simple median filter[24] could be used. However, this is rarely the case. Most common is the fact that the background image has to be adjusted as the time pass by. Huang et al.[9] address the problem of separating foreground from background in color images. They assume that background regions are relatively smooth but may have gradually varying colors or slightly textured. Voting may be another strategy to define the background pixel value from a set of samples. 


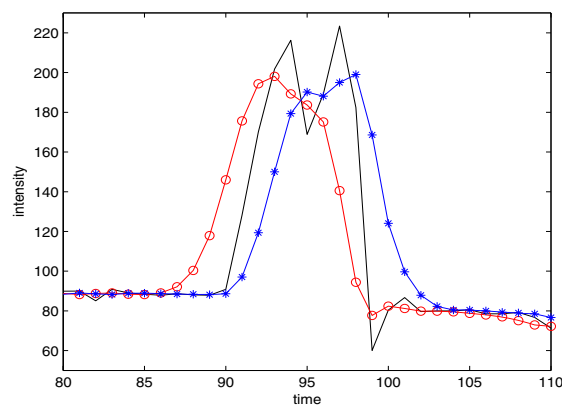

(a) Intensity.

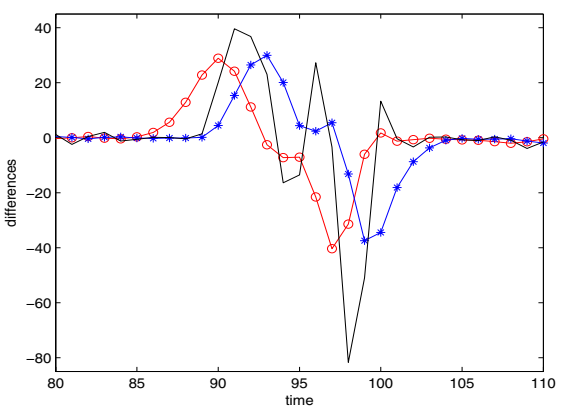

(b) Differences.

Fig. 3. Convolution with a Half-Gaussian that takes into account only the values before the current position (red-circled line) or the values after the current position (blue-starred line)

For a given pixel, Tai and Song [20] propose to accumulate the intensity value observations into a histogram. The most frequent value is used as an estimate of the background value. They apply the proposed method to a vision-based traffic monitoring system to segment moving vehicles from traffic image sequences. In a seminal paper, Stauffer and Grimson[19] proposed to model each pixel as a mixture of Gaussians. The gaussian distributions of the adaptive mixture model are evaluated to determine which are most likely to result from a background process. Each pixel is classified based on whether the Gaussian distribution which represent it most effectively is considered part of the background model. This model has been adapted widely, and studied extensively. For instance, Wayne and Schoonees[15] developed a tutorial paper to describe a practical implementation of the Stauffer-Grimson algorithm and provide values for all model parameters. In their document, they show what approximations to the theory were made and how to improve the standard algorithm by redefining those approximations.

In the rest of the paper, we introduce a technique to detect moving objects in a scene with static background imaged from a fixed camera. We focus on the background update stage, assuming that initialization, shadows, uncovered background, and region and frame level analysis can be deal with using some of the techniques mentioned before. In 12 , we review the intrinsic curve paradigm and illustrate how it is useful to detect moving boundaries. Then, in $\$ 3$, the background detection approach using mixture of Gaussians is visited. There, we show how both ideas can be blended. Next, in $\$$, we present experiments with several image sequences under an ample set of conditions. Finally, we summarize our results and outline future research.

\section{Detecting Moving Boundaries}

In this section, it is introduced an algorithm to distinguish, at pixel level, moving from static surfaces by analyzing a pixel dynamics in a particular intrinsic curve space. 


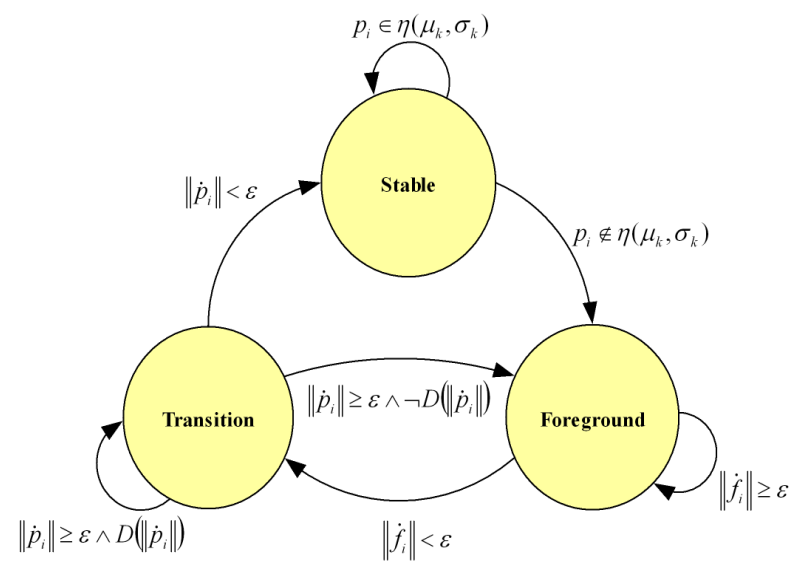

Fig. 4. State diagram for a pixel. The conditions to change state are commented within the text.

\subsection{Intrinsic Curves}

In the context of stereovision, Tomasi and Manduchi [21] proposed the description of scanlines in terms of a set of operators $M_{n}$. Thus, a intrinsic curve is a curve $\mathbf{m}(t)=\left\{m_{n}(t), t \in \mathbf{R}\right\}$ in $\mathbf{R}^{N}$ parameterized by the real variable $t$ such that $\mathbf{m}$ is generated by applying $N$ operators $M_{n}$ to the original signal $m_{0}(t)$ to produce the new signals $m_{n}(t)$. Tomasi and Manduchi were specially interested in diffeomorphisms, $i$. e., mappings between manifolds which are differentiable and have a differentiable inverse 23]. In particular, as Tomasi and Manduchi did in their paper, in this study is interesting to apply operators of the form

$$
m_{n}(t)=\left[M_{n} m_{0}\right](t)=\frac{d^{n}}{d t^{n}} m_{0}(t) .
$$

Let us focus on a single pixel intensity dynamics throughout time. Suppose that it has a background intrinsic curve model $\alpha(t)$ while $\beta(t)$ is the intrinsic curve for the values being observed. Tomasi and Manduchi noted that in the intrinsic curve space, noise apart, both curves will follow the same trajectory. However, during an occlusion, they will experience a significant deviation of one respect to the other. When the occlusion ends, both curves will meet again in a place that depends on whether the occluding object became part of the background. This idea is illustrated in Fig. 1. In general, an intrinsic curve that belongs to the background is defined within what we call a stability zone. Here, stable corresponds to the notion that the observed curve values are drawn from a stable distribution[14]. For simplicity, we assume that the stable distribution follows a Gaussian model given by

$$
h(\mathbf{x})=\frac{1}{(2 \pi)^{N / 2}\|\Sigma\|^{1 / 2}} \exp \left[-\frac{1}{2}(\mathbf{x}-\mu)^{T} \Sigma^{-1}(\mathbf{x}-\mu)\right],
$$

with $\mathbf{x}=\mathbf{m}(t)$, the observed intrinsic curve, and $\mu=\left[\bar{m}_{0}, \ldots, \bar{m}_{N-1}\right]$, the intrinsic curve components mean value, and covariance matrix $\Sigma$. Background occlusions due 
to foreground objects are rather difficult to describe in terms of a parametric distribution. Intrinsic curves help us to distinguish between both.

We have noted three kinds of intrinsic curves: leaving, returning, and steady. The leaving case occurs when the foreground objects integrates into the background (illustrated in Fig 2(a)-(c)). This is the typical case, for instance, of a car parking. Imagine that a intrinsic curve is in the stability zone. When the car occludes the background the curve departs from it. Then, when the car finally parks, the curve finds, in general, another stability zone and the parked car is integrated into the background model. In the returning case (illustrated in Fig 2(d)-(f)), the curve remains stable until an object occludes the background. At that moment, the curve leaves the stability zone. When the object passes, the curve comes back to its original stability zone. The previous two cases occur when the background is occluded. The steady case describes the long term behavior of a pixel that belongs to the background. This event occurs, for instance, when the illumination changes smoothly as the day passes by. Fig. 2 (g)-(i) show two days of observation of the same pixel location. As the day passes by the illumination increases and decreases smoothly.

\subsection{Detecting Motion}

To detect moving boundaries using intrinsic curves, the prime problem is to distinguish the stability zone. The pixel will be classified as being part of the background until it leaves the stability zone, and during that time it will be part of the foreground class. It will return to the background class once it stabilizes again. In practice, image noise makes it hard to classify the state of a pixel. Let the pixel intensity $m_{0}(t)$ at time $t$ be described by $m_{0}(t)=n(t)+\xi(t)$, where $n(t)$ is the true image intensity value and $\xi(t)$ is the image noise. Traditionally, one way to remove this noise has been by using low pass filters. However, applying filters across region boundaries may result in undesirable results because regions belong to different populations. To remove noise, a zero-mean Gaussian filter is applied but with a twist. Half-Gaussian are used to remove the noise, such that operators

$$
g_{p}(t)=\left\{\begin{array}{cc}
\sqrt{\frac{2}{\pi \sigma^{2}}} \exp \left(\frac{t^{2}}{2 \sigma^{2}}\right)-\infty<t \leq 0 \\
0 \quad \text { otherwise }
\end{array}, \text { and } g_{f}(t)=\left\{\begin{array}{ll}
\sqrt{\frac{2}{\pi \sigma^{2}}} \exp \left(\frac{t^{2}}{2 \sigma^{2}}\right) & 0 \leq t<\infty \\
0 & \text { otherwise }
\end{array},\right.\right.
$$

are applied simultaneously. The original signal $m_{0}(t)$ is thus filtered out resulting in two estimates, $p_{0}(t)$ and $f_{0}(t)$, that take into account only frames before and after the current one, respectively, such that

$$
p_{0}(t)=m_{0}(t) * g_{p}(t), \quad \text { and } \quad f_{0}(t)=m_{0}(t) * g_{f}(t) .
$$

Let $m(k)$ correspond to the observation of a pixel in position $\mathbf{x}$ at time $k$. The sequence is smoothed using as many frames as required by the filter window width. Let us call $k$ the present time. Half of the filter's window include values corresponding to the previous frames and half of it values corresponding to the next frames. After smoothing, there will be signals $p_{0}(k)$ and $f_{0}(k)$ corresponding to estimates of the true pixel intensity. Intrinsic curve descriptors for the $i$-order difference can be computed using the estimates of the $(i-1)$-order. This way, there will be an intrinsic curve description that 
considers the previous frames, $\mathbf{p}(k)=\left\{p_{0}(k), p_{1}(k), \ldots, p_{N-1}(k)\right\}$, and another considering the next ones, $\mathbf{f}(k)=\left\{f_{0}(k), f_{1}(k), \ldots, f_{N-1}(k)\right\}$. As it is illustrated in Fig. 3. $\mathbf{p}$ and $\mathbf{f}$ are useful to detect respectively the beginning and ending of an occlusion.

\section{Adding Memory}

Stauffer and Grimson[19] proposed one of the most popular methods to estimate the model of the intensity variations for an image sequence took from a fixed camera. Their method is based on computing on-line the parameters of a mixture of Gaussians (MOG) for each individual pixel. In this method, a given pixel intensity value is classified as either part of the background or the foreground depending on whether the value is likely to be interpreted by the respective statistical model. In practice, the method generates between 3 and 5 Gaussians. Intrinsic curves aims to improve this model in two ways. On the one hand, they do not feed the mixture of Gaussians with foreground pixels. On the other hand, they take into account not just the scalar pixel value but a richer description of the pixel's dynamics that carries information about the interpixel variation. In this section, we complement the intrinsic curve model with the addition of memory capabilities that could be used to describe what has been occurring in the past. First, the MOG model is described. Then, MOG and intrinsic curves are combined into a single paradigm.

\subsection{Mixture of Gaussians}

MOG aims to model the image of a dynamic scene as perceived from a fixed camera by a set of Gaussians. Given a set of $n$ points in one dimension, $x_{1}, \ldots, x_{n} \in \mathbf{R}$, and a family $\mathcal{F}$ of probability density functions on $\mathbf{R}$, the problem is to find the probability density $f(x) \in \mathcal{F}$ that is most likely to have generated the given points. In this method, each member of the family $\mathcal{F}$ has the same general Gaussian form. Each member is distinguished by different values of a set of parameters $\theta$. That is

$$
f(x ; \theta)=\sum_{k=1}^{K} q_{k} g\left(x ; \mu_{k}, \sigma_{k}\right), \quad \text { where } \quad g\left(x ; \mu_{k}, \sigma_{k}\right)=\frac{1}{\sqrt{2 \pi} \sigma_{k}} \exp \left[-\frac{1}{2}\left(\frac{x-\mu_{k}}{\sigma_{k}}\right)^{2}\right],
$$

is a 1 -dimensional Gaussian function and $\theta=\left(\theta_{1}, \ldots, \theta_{K}\right)=\left(\left(q_{1}, \mu_{1}, \sigma_{1}\right), \ldots,\left(q_{K},-\right.\right.$ $\left.\left.\mu_{K}, \sigma_{K}\right)\right)$, is a $3 K$-dimensional vector containing the mixing probabilities $q_{k}$ as well as the means $\mu_{k}$ and standard deviations $\sigma_{k}$ of the $K$ Gaussian functions in the mixture. When a new observation $x_{t}$ is available, it is compared again the parameters of the Gaussian models. If $\left\|x-\mu_{k}\right\| \leq \alpha \sigma_{k}$, then it is assumed that the observation is likely to be produced by a perturbation of the true value similar to the one expressed by the $k$-model. Typically, $\alpha$ is chosen to be 3, meaning that $x$ is within $99.73 \%$ of the cases occurring under this model. If an observation occurs that can not be explained by the current set of Gaussians, a new model with high variance and centered around the observation is initialized. This model becomes part of the mixture of Gaussians. Otherwise, the new observations helps to learn the true value of the parameters. Most commonly the learning process followed is called Estimation-Maximization (EM). In the first place a lower bound function $b_{i}(\theta)$ is claimed to approximate $f$. Then the parameters that 
maximize this function are found. Since not all the observations are available at once, an on-line version of the EM algorithm uses the following set of equations.

$$
\mu_{t+1}=\rho \mu_{t}+(1-\rho) x_{t}, \quad \text { and } \quad \sigma_{t+1}^{2}=\rho \sigma_{t}^{2}+(1-\rho)\left(x_{t}-\mu_{t+1}\right)^{2},
$$

where $\rho \in[0,1]$ is the learning rate.

\subsection{Estimating the Background Model}

In our model, a pixel can be in anyone of three states (see Fig.4): stable, transition, and foreground. To reduce the memory space and computing demands, let $\mathbf{p}(k)=\left(p_{k}, \dot{p}_{k}\right)$ and $\mathbf{f}(k)=\left(f_{k}, \dot{f}_{k}\right)$ be respectively the estimate for the intrinsic curves for a particular pixel at time $k$ using the samples before and after the current frame. Initially, a pixel is classified in the transition state. The change of state is governed by the following rules:

- A transition pixel will stay like that if $\left\|\dot{p}_{k}\right\|>\epsilon$, and $\left\|\dot{p}_{k}\right\|$ is showing a decreasing tendency. Otherwise, if $\left\|\dot{p}_{k}\right\|>\epsilon$ but $\left\|\dot{p}_{k}\right\|$ does not show a decreasing tendency the pixel will change state to foreground. A transition pixel will change its state to stable if $\left\|\dot{p}_{k}\right\|<\epsilon$.

- An stable pixel will remain in that state provided $\mathbf{p}$ is in the stability zone. Otherwise, the state will change to foreground.

- A foreground pixel will stay like that if $\mathbf{f}$ is not in the stability zone. Otherwise, its state will change to transition.

The decreasing tendency function $D\left(x_{i}\right)$ is used to solve the problems caused by temporal aliasing. When an object has moving parts that occlude and accretes the background too fast, the noise filters receive samples from different distributions and the result is not accurate. A similar situation occurs when objects move too close to each other. The filter's window spans both objects mixing up their respective populations. However, in those cases $\mathbf{f}$ returns for a moment to a stability zone and then bounces out in response to the following occluding object. If during this time $\mathbf{p}$ keeps approaching the stability zone then it can be assumed that the pixel is a stable one.

A pixel classified as stable is not necessarily part of the background. That is, imagine that a large, homogeneous object is moving on top of a static background. The frontal part of the object will be detected as a moving region as well as the rear part. However, once a pixel enters the stable state, it will start building a model for the variation of the intensity of this pixel. What is being produced is a set of layers that model the pixel dynamics. So the criteria that we have used is the following one. If there is a pixel with more that one Gaussian description and one of the Gaussians has been used in the current frame, i.e., the pixel is in the stable state, then that pixel is considered part of the foreground. This strategy has proved to be useful for instance when detecting leftover objects. Imagine for instance a parking lot. When a new car parks, its figure outstand because although it stabilizes, a different background was there before. A consequence of this is that when a parked car leaves, its silhouette will remain. So it really depends on what was the previous state of the scene.

Aging can be applied for pixels that have been in the scene for a long time, but whose Gaussian description has not been visited for some time, the application erases 
the Gaussian that models it. This allows to eliminate the cases described before where parked objects leave the scene and left their ghost behind. Also, Gaussians with few samples not used for some time are eliminated from the Gaussian mixture because it is considered that they were the result of noise.

\section{Experimental Results}

The model just described was implemented on an Intel Pentium IV computer with $3 \mathrm{GHz}$ clock frequency. It was programmed in MATLAB and tested with several sequences that included indoors and outdoors scenarios, with natural and artificial light, imaged during nighttime and daytime. For the experiments, color images were converted into grayscale ones. The average processing speed was about 5,300 pixels per second. Due to lack of space, we illustrate the results with a few frames out of a poll of sequences.

PETS stands for Performance Evaluation of Tracking and Surveillance Systems [3] (first row in Fig. 5). The sequence has 2688 JPEG frames. The individual frame resolution is $768 \times 576$ color pixels. There are seven persons or groups of persons that at different times cross the scene. There are also three vehicles that park or do some kind of manoeuver, like moving back and forth. In general, when the objects move their shape is well delineated. In this sequence, the moving objects either pass by or arrive to the scene. There is one truck that enters the scene, parks, and afterwards leaves the scene. In such cases, the foreground objects are correctly detected and when they leave aging and small groups filtering can get ride of the traces left. However, see the Laboratory sequence, which has 182 color frames with resolution $640 \times 480$ (second row in Fig. 5). In this sequence a person walks around a computer laboratory. The person dresses a shirt with a black squares drawing and black pants. The sequence starts with the person standing at the door. As he leaves this position, his silhouette is left behind. Whether to leave or remove one of such figures depends on a decision that cannot be taken at pixel level and also on factors such as the initial state of the scene background and ultimate application of the surveillance system. Fig.5(b.1) shows also the trajectory followed by the person. This trajectory was computed using the blob centroid in each frame of the sequence. Some problems related to shadows that appear in this sequence are more evident in the sequence Soccer. The Soccer sequence has 9,744 frames with resolution $320 \times 240$ (third row in Fig. 5). It shows a nocturne soccer game where 22 players, a referee, the ball and the public provide a quite dynamic scenario. The camera was placed about 80 meters from middle field. The illumination conditions, dominated by neon lamps, make the objects in the scene to cast multiple shadows. The shadows is the regular price that has to be paid for processing is grayscale instead of color. Still, in this scene moving objects are well detected even considering their size and the type of illumination. Another experiment shows the Crossroads sequence, which was took from a 28 meters high tower placed in one of the corners of a crossroads (Fig. 5). From that viewpoint is possible to cover the whole intersection. The sequence has 2000 frames, each one with resolution $320 \times 240$. This sequence is very challenging because the vehicles become part of the background and then, after a while, they suddenly start moving again, at high speed. So the background has to recover quickly but there are other vehicles passing by. Also, long buses with homogeneous roofs are 


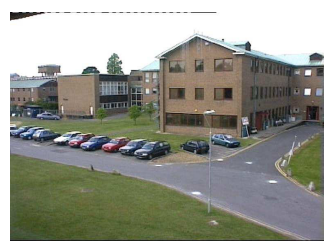

(a.1) Scene.

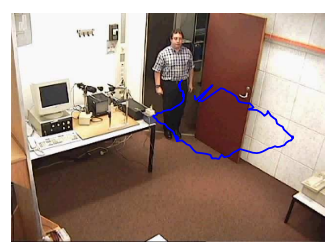

(b.1) Scene.

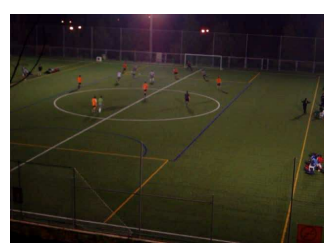

(c.1) Scene.

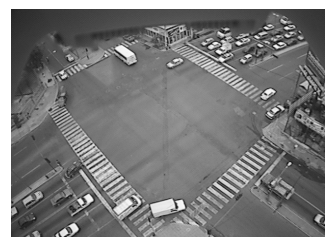

(d.1) Scene.

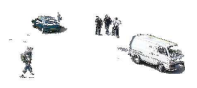

(a.2) Frame 950.

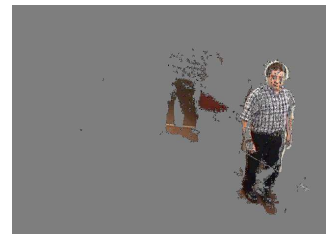

(b.2) Frame 058.

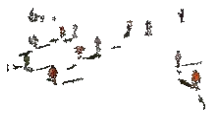

(c.2) Frame 4000.

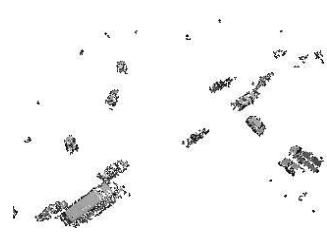

(d.2) Frame 72. (a.3) Frame 2610.

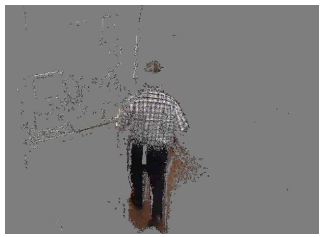

(b.3) Frame 118.

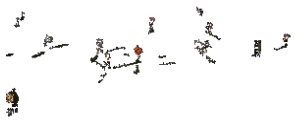

(c.3) Frame 8000.

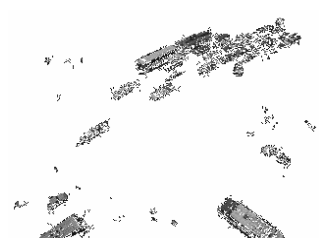

(d.3) Frame 742.

Fig. 5. Experimental results

detected correctly. In general, depending on what is initially consider to be part of the background, our method can be used to detect left over objects, which could leave the scene at a later stage.

\section{Conclusion}

In this document, we have shown how an intrinsic curves could be used to detect accretion and occlusion regions in a scene containing moving objects. Using, this technique alone will produce fragmented segments for large objects with homogeneous texture. Hence, Gaussian mixture models provide an ideal vehicle to preserve information about the recent pixel intensity dynamics. Our algorithm shows good results for updating the model of the background under a variety of scenarios that include indoors and outdoors 
environment in a number of different illumination conditions. It has been noted that depending on the initial background state and ultimate application, the algorithm is able to keep track of leftover objects. This is an important property that may be useful for security applications. As stated, the algorithm is based on the grayscale processing of individual pixels without regard of the behavior of the local neighborhood. Color information, and hierarchical analysis may be prove to be helpful to eliminate shadows, detect camouflage and sudden global illumination changes.

\section{References}

1. Shao-Yi Chien, Shyh-Yih Ma, and Liang-Gee Chen. Efficient Moving Object Segmentation Algorithm using Background Registration Technique. Circuits and Systems for Video Technology, IEEE Transactions on, 12(7):577 - 586, 2002.

2. R. Cucchiara, C. Grana, M. Piccardi, and A. Prati. Detecting Moving Objects, Ghosts, and Shadows in Video Streams. IEEE Transactions on PAMI, 25(10):1337 - 1342, 2003.

3. James Ferryman. Performance Evaluation of Tracking and Surveillance On-Line Evaluation Service. http://visualsurveillance.org/PETS2001, February 2006.

4. P. R. Giaccone, D. Tsaptsinos, and G. A. Jones. Foreground-Background Segmentation by Cellular Neural Networks. In ICPR, volume 2, pages 438 - 441, 2000.

5. D. Gutchess, M. Trajkovics, E. Cohen-Solal, D. Lyons, and A. K. Jain. A Background Model Initialization Algorithm for Video Surveillance. In ICCV, volume 1, pages 733 - 740, 2001.

6. Hongzhe Han, Zhiliang Wang, Jiwei Liu, Zhengxi Li, Bin Li, and Zhongtao Han. Adaptive Background Modeling with Shadow Suppression. In Intelligent Transportation Systems, Proceedings IEEE, volume 1, pages 720 - 724, 2003.

7. Yinghua He, Hong Wang, and Bo Zhang. Updating in Illumination-Variant Scenes. In Proceedings IEEE Intelligent Transportation Systems, pages 515 - 519, 2003.

8. T. Horprasert, D. Harwood, and L. S. Davis. A Robust Background Subtraction and Shadow Detection. In Proceedings of the Fourth Asian Conference on Computer Vision, 2000.

9. Qian Huang, B. Dom, N. Megiddo, and W. Niblack. Segmenting and Representing Background in Color Images. In ICPR, volume 3, pages 13 - 17, 1996.

10. A. Kaup and T. Aach. Efficient Prediction of Uncovered Background in Interframe Coding using Spatial Extrapolation. In IEEE International Conference on Acoustics, Speech, and Signal Processing, volume 5, pages 501-504, 1994.

11. P. Kumar, S. Ranganath, and W. Huang. Queue Based Fast Background Modelling and Fast Hysteresis Thresholding for Better Foreground Segmentation. In Multimedia. Proceedings of the 2003 Joint Conference of the Fourth International Conference on, volume 2, pages 743 $-747,2003$.

12. Horng-Horng Lin, Tyng-Luh Liu, and Jen-Hui Chuang. A Probabilistic SVM Approach for Background Scene Initialization. In ICIP, volume 3, pages 893 - 896, 2002.

13. K. E. Matthews and N. M. Namazi. A Bayes Decision Test for Detecting UncoveredBackground and Moving Pixels in Image Sequences. IEEE Transactions on IP, 7(5):720 $-728,1998$.

14. John P. Nolan. Stable Distributions. http://academic2.american.edu/jpnolan/stable/chap1.pdf, February 2006.

15. Johann A. Schoonees P. Wayne Power. Understanding Background Mixture Models for Foreground Segmentation. In Proceedings Image and Vision Computing New Zealand, page 267, 2002.

16. M. Piccardi. Background Subtraction Techniques: A Review. In IEEE ICSMC, volume 4, pages $3099-3104,2004$. 
17. R. Pless, J. Larson, S. Siebers, and B. Westover. Evaluation of Local Models of Dynamic Backgrounds. In CVPR, pages 73-78, 2003.

18. R. J. Qian and M. I. Sezan. Video Background Replacement without a Blue Screen. In ICIP, pages 143-146, 1999.

19. C. Stauffer and W. E. L. Grimson. Adaptive Background Mixture Models for Real-Time Tracking. In CVPR, volume 2, pages 246 - 252, 1999.

20. Jen-Chao Tai and Kai-Tai Song. Background Segmentation and its Application to Traffic Monitoring using Modified Histogram. In Networking, Sensing and Control, 2004 IEEE International Conference on, volume 1, pages 13 - 18, 2004.

21. Carlo Tomasi and Roberto Manduchi. Stereo Matching as a Nearest-Neighbor Problem. IEEE Transactions on PAMI, 20(3):333-340, 1998.

22. K. Toyama, J. Krumm, B. Brumitt, and B. Meyers. Wallflower: Principles and Practice of Background Maintenance. In ICCV, volume 1, pages 255 - 261, 1999.

23. Eric W. Weisstein. Diffeomorphism. From MathWorld-A Wolfram Web Resource. http://mathworld.wolfram.com/Diffeomorphism.html, September 2005.

24. Yufang Zhang, P. Shi, E. G. Jones, and Q. Zhu. Robust Background Image Generation and Vehicle 3D Detection and Tracking. In IEEE Conference on Intelligent Transportation Systems, pages $12-16,2004$.

25. Yue Zhou and Hai Tao. A Background Layer Model for Object Tracking through Occlusion. In $I C C V$, volume 2, pages 1079 - 1085, 2003. 